\title{
A Rare Case of PEComa of the Liver
}

\author{
Abigail Attard $^{\mathrm{a}}$ Naomi Piscopo $^{\mathrm{a}}$ John Schembri ${ }^{\mathrm{a}}$ Tiffany Buhagiar ${ }^{\mathrm{b}}$ \\ Kelvin Cortis ${ }^{c}$ Pierre Ellul ${ }^{a}$

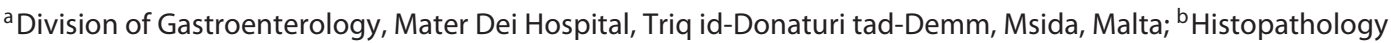 \\ Department, Mater Dei Hospital, Triq id-Donaturi tad-Demm, Msida, Malta; ${ }^{\mathrm{C}}$ Medical Imaging, Hepatobiliary \\ Radiology and Interventional Oncology, Mater Dei Hospital, Triq id-Donaturi tad-Demm, Msida, Malta
}

\section{Keywords}

Hepatic angiomyolipoma $\cdot$ PEComa $\cdot$ Differential diagnosis

\section{Um caso raro de PEComa hepático}

\section{Palavras Chave}

Angiomiolipoma hepático · PEComa · Diagnóstico diferencial

\section{Introduction}

Perivascular epithelioid cell tumors (PEC) are benign cells with multiple differentiation potential capable of differentiating into the vascular smooth muscle and epithelial cells. Primary hepatic angiomyolipomas (HAMLs), a subset of PEComas, are extremely rare, have a higher incidence in females, and are usually asymptomatic. The natural history of HAMLs is not well-established or predictable yet. Correct preoperative diagnosis of HAML is reported to be less than $25 \%$ [1].

\section{Case Presentation}

A 69-year-old Caucasian male with no significant past medical history, presented with non-specific abdominal discomfort, which had been going on for more than 6 weeks. There were no change in bowel habit and no urinary symptoms. Abdominal examination was unremarkable. Blood investigations including complete blood count, renal profile, liver profile, and the inflammatory markers C-reactive protein and erythrocyte sedimentation rate were within normal ranges.

In view of his persistent symptoms, an abdominal computed tomography (CT) was performed. This demonstrated a $4.5 \times 3.3$ $\mathrm{cm}$ hypodense solid mass in segment VI of the liver (Fig. 1), which had an ill-defined border and was mildly hypovascular. The lesion demonstrated complete circumferential enhancement on the portal venous phase and remained hypodense with respect to the rest of the liver parenchyma on the excretory phase. These findings are not typical of a hemangioma.

The lesion was further characterized with a magnetic resonance (MR) scan of the liver. Our MR examinations were performed using a super-conducting magnet operating at $3 \mathrm{~T}$ (Philips Ingenia; Philips Medical Systems, Best, The Netherlands) using a phased array body coil centered on the liver. The anatomic region covered extended from the lung bases to the lower pole of the right kidney. MR imaging was performed before and after Gd-EOB (gadoxetate disodium injection $0.25 \mathrm{mmol} / \mathrm{mL}$, Primovist ${ }^{\circledR}$; Bayer, Leverkusen, Germany). Contrast was administered at $0.1 \mathrm{mmol} / \mathrm{kg}, 2.4$ $\mathrm{mL} / \mathrm{s}$ through an 18-to-21-gauge peripheral intravenous cannula using an automated dual-chamber injector (Spectris MR Injector; Medrad, Pennsylvania, PA, USA).

\section{2020 Sociedade Portuguesa de Gastrenterologia} Published by S. Karger AG, Basel

This article is licensed under the Creative Commons AttributionNonCommercial-NoDerivatives 4.0 International License (CC BYNC-ND) (http://www.karger.com/Services/OpenAccessLicense). Usage and distribution for commercial purposes as well as any distribution of modified material requires written permission.
Abigail Attard

Division of Gastroenterology

Mater Dei Hospital, Triq id-Donaturi tad-Demm

MT-MSD2090 Msida (Malta)

abigail.attard@gov.mt 
1

Fig. 1. CT image of PEComa in segment VI of the liver.

Fig. 2. Coronal T2-weighted image showing a well-defined heterogeneously hyperintense lesion in the medial aspect of the right liver lobe.
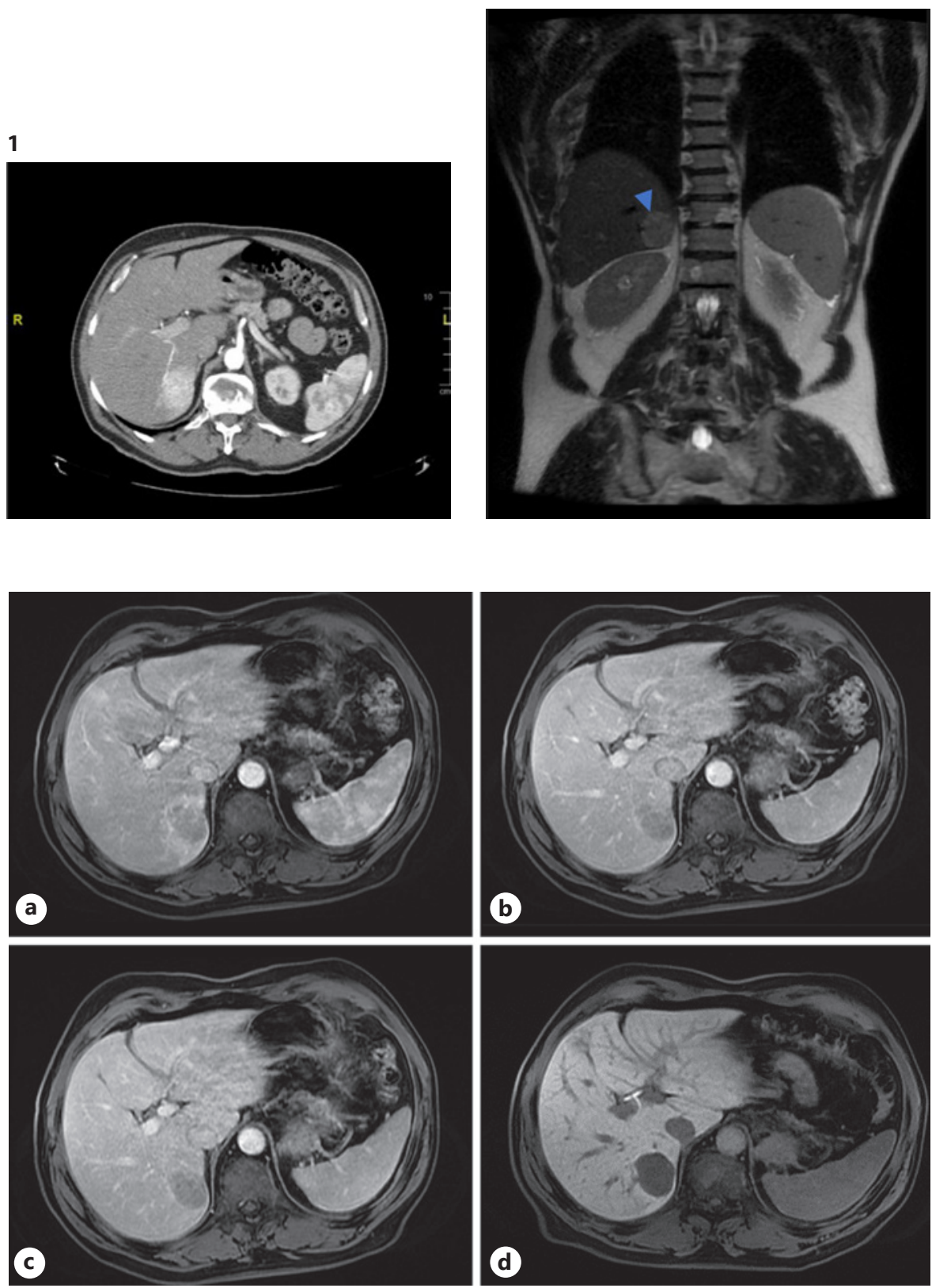

Fig. 3. Post-contrast 3D. T1-weighted axial fat-suppressed images showing a perfusion anomaly surrounding this lesion. This lesion remains hypovascular on arterial phase (a) and portal/late venous phase (b, c) imaging. No uptake of hepatobiliary contrast media is seen on 20-min delayed hepatobiliary phase imaging (d).

T2 imaging demonstrated a well-defined heterogeneously hyperintense lesion in the medial aspect of the right liver lobe (Fig. 2). Post-contrast 3D T1-weighted fat-suppressed images showed a perfusion anomaly surrounding this lesion. It remained hypovascular on arterial phase (Fig. 3a) and portal/late venous phase (Fig. 3b, c) imaging. No uptake of hepatobiliary contrast media was seen on 20-min delayed hepatobiliary phase imaging (Fig. 3d). Additionally, the lesion demonstrated mild restriction of diffusion, as confirmed on the apparent diffusion coefficient (ADC) map (Fig. 4).

In view of the broad differential diagnosis, the lesion was biopsied, and histology demonstrated a clear-cell neoplasm with immunostaining, suggesting a perivascular epithelioid cell tumor, a
HAML, the neoplastic elements being positive for Melan-A, HMB45 , vimentin, and smooth muscle actin (SMA) (Fig. 5, 6).

The patient was further assessed by means of an esophagogastroduodenoscopy, colonoscopy, and a skin and ocular check for any potential primary lesions. These were all normal. A PET (positron emission tomography)-CT (Fig. 7) that was performed demonstrated increased uptake by the liver lesion, thus supporting the risk of malignant potential.

In view of potential malignant features on imaging and the non-diagnostic features on imaging and histology, the lesion was resected. After surgery, the lesion was consistent with a HAML. Twenty months later, the patient is still in remission with imaging consistent with the previous surgical resection. 

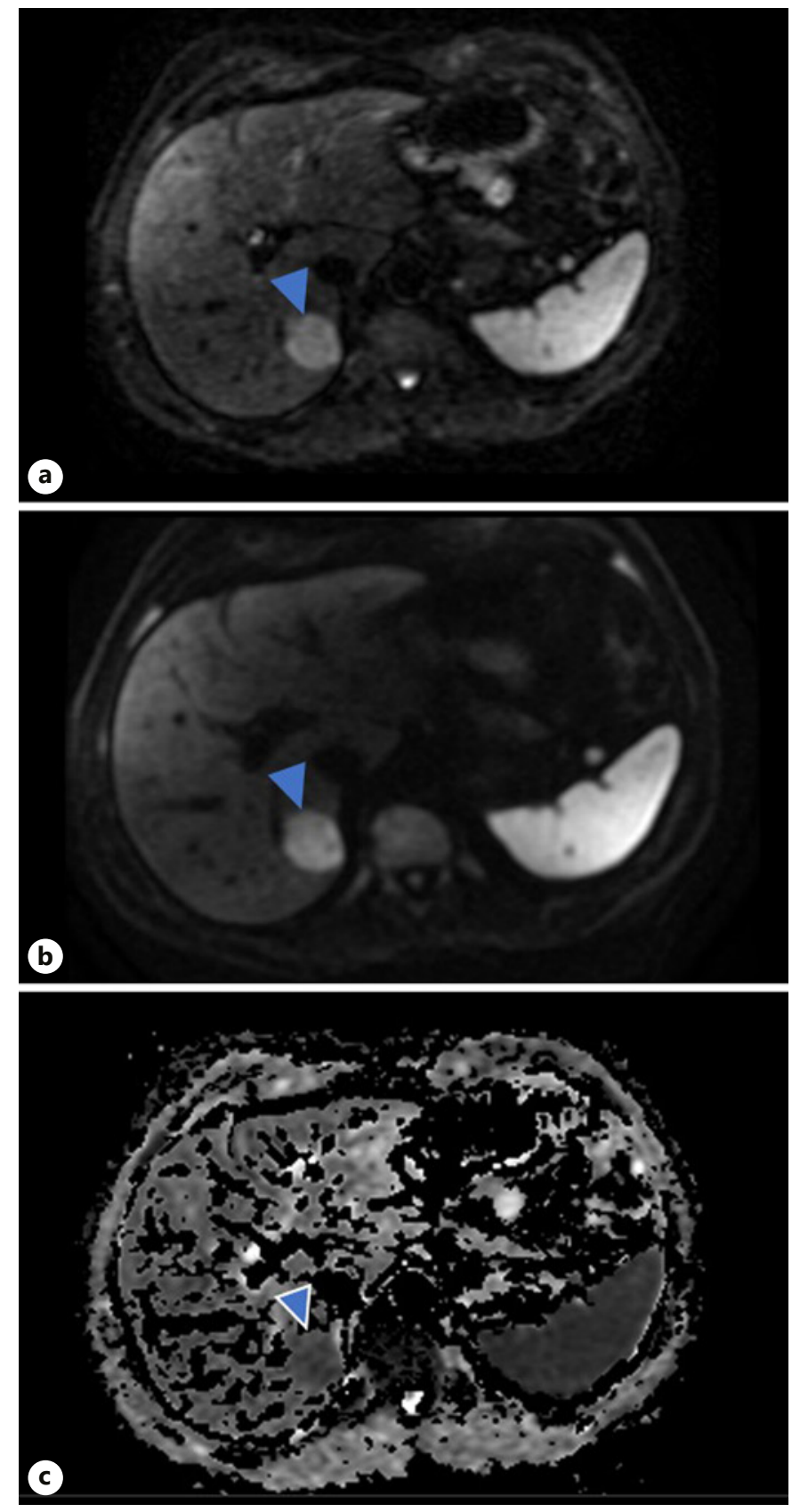

Fig. 4. Diffusion-weighted imaging. Top image: $b=50$ (a). Middle image: $b=800$ (b). Bottom image: ADC map (c). The lesion is evident on $b=50$ and slightly brighter on $b=800$. This is compatible with mild restriction of diffusion, as confirmed on the ADC map.

\section{Discussion and Conclusion}

Most PEComas exhibit benign characteristics, but aggressive PEComas demonstrating potentially invasive growth with malignant potential and metastatic risk have

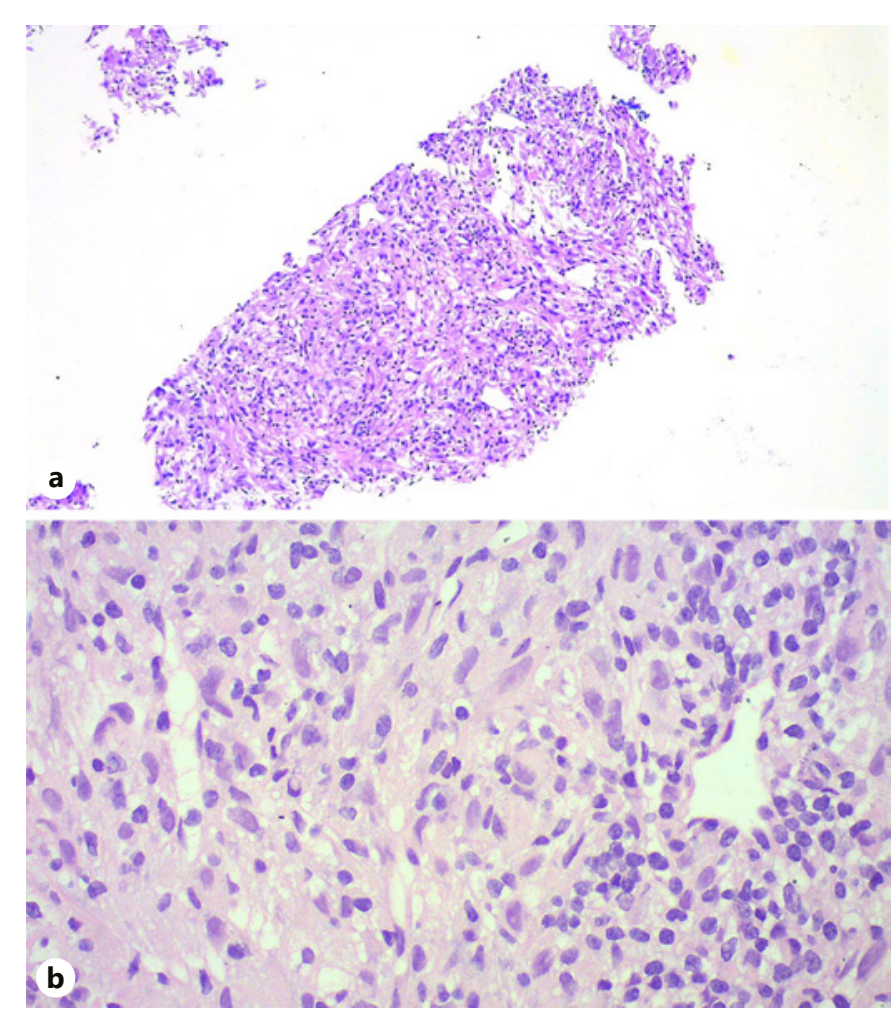

Fig. 5. Pathological findings of the PEComa. a H\&E. Low-power view $(\times 100)$. b H\&E. High-power view $(\times 400)$. A neoplastic proliferation composed of spindle and polygonal cells with clear to pale eosinophilic cytoplasm, arranged in a haphazard pattern around small vascular spaces.

been reported. Malignant transformation is more likely with tumor sizes $>5 \mathrm{~cm}$, infiltrative growth pattern, high nuclear grade, mitotic activity $>1$ per 50 high-power fields, and evidence of necrosis [2].

HAMLs are a subset of PEComas. They are extremely rare, have a higher incidence in females, and are usually asymptomatic. The majority of HAMLs occur in adult females of Asian origin [1]. These tumors are made of three main tissue components: blood vessels, epithelia, or spindle-shaped smooth muscle cells, and mature adipose tissue [3]. Angiomyolipomas are most frequently found in the kidney, and $6-10 \%$ of the patients with HAMLs suffer from tuberous sclerosis $[4,5]$. The occurrence of a HAML is usually in asymptomatic patients, with the HAML being detected incidentally [3].

Features of a HAML on radiological studies can be non-specific. Other possible diagnoses for such a lesion on imaging include hepatocellular carcinoma (HCC), cholangiocarcinoma, gastrointestinal stromal tumor, ad- 


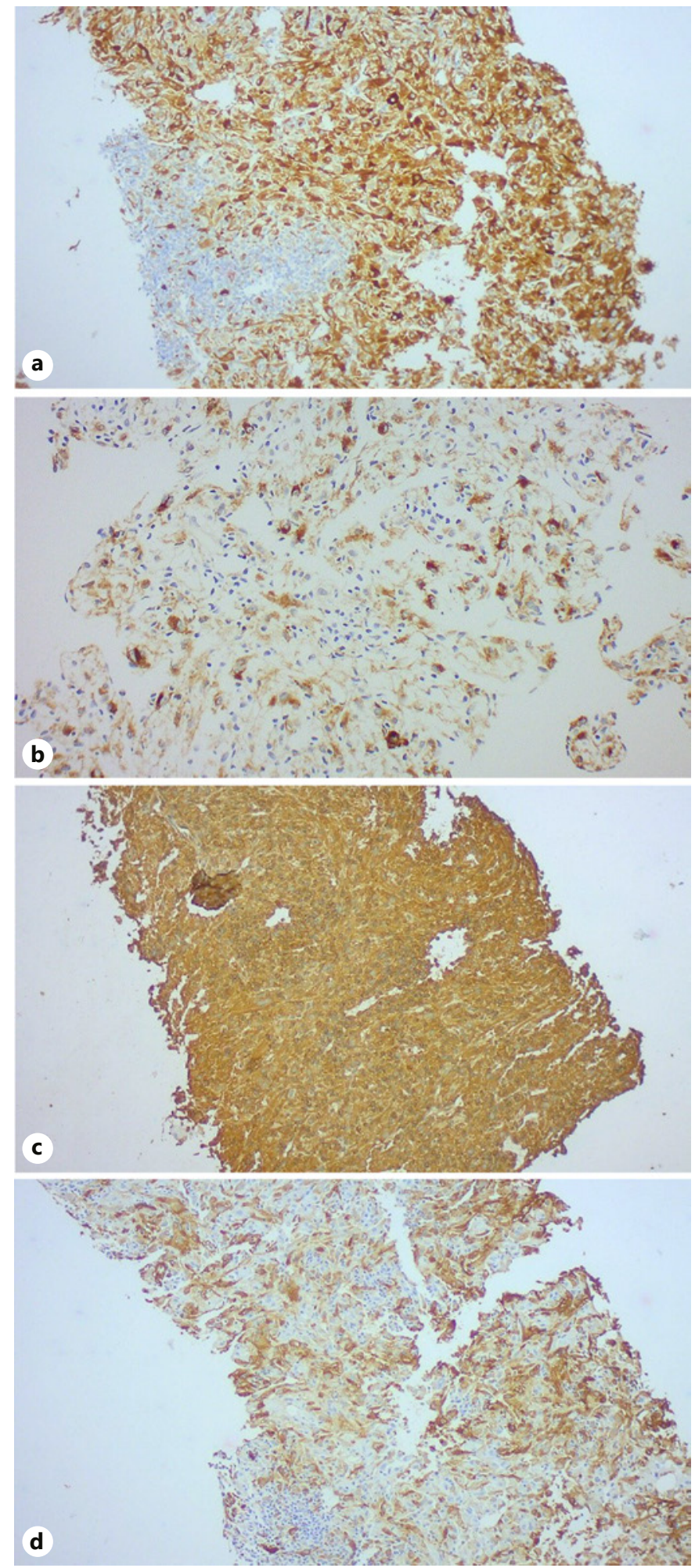

Fig. 6. Immunohistochemistry staining of PEComa. The neoplastic cells express Melan-A (a), HMB-45 (b), vimentin (c), and SMA (d).

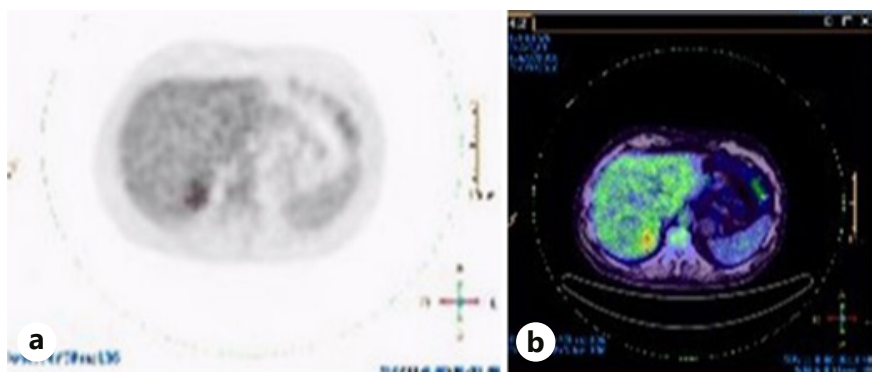

Fig. 7. PET-CT showing increased uptake in segment VI of liver.

enoma, leiomyoma, melanoma, angiosarcoma, lipoma, liposarcoma, and hemangioma $[1,6]$.

HAMLs typically contain fat. However, the radiological features of such a lesion are determined by the relative amount of adipose cells within it. MR is more sensitive than CT in detecting adipose tissue. The high-signal-intensity foci on T1-weighted images are likely to be due to abundant lipomatous tissue. Lipomatous lesions may also be determined by the chemical shift imaging technique. Diagnostic difficulties arise between HAMLs and other liver tumors comprising adipose tissue. These include fatty metamorphosis of HCC, hepatocellular adenoma with fat content, lipoma, and liposarcoma [3, 7].

In the presented case, MR did not demonstrate intralesional fat, which is relatively exceptional to a HAML [3]. The lesion was hyperintense on T2 and hypointense on T1, thus making it difficult to distinguish from a HCC, which typically does not include intralesional fat.

There are four microscopic pathological subclassifications of HAMLs. The subtypes depend on the proportion of each tissue type, specifically the dominant tissue, within the tumor: (I) hybrid type: commonest type, consisting of equal estimates of each tissue component, that is, smooth muscle cells, blood vessels, and adipose tissue; (II) myoma type: primarily consisting of smooth muscle cells; (III) lipoma type: having a predominance of adipose tissue; and (IV) hemangioma type: mainly consisting of blood vessels [1].

Histological diagnosis of HAMLs might be challenging in view of the pleomorphism and heterogeneity of the histological features of HAMLs as well as because of the rarity of HAMLs themselves. Several characteristics in a HAML might be mistaken for features of a HCC: polygonal cells in trabecular arrangement, peliosis, nuclear pleomorphism, prominent eosinophilic nucleoli, deficient reticulin framework, presence of glycogen, eosinophilic globules, and tumor necrosis [8]. 
Diagnostic specificity for HAMLs is enhanced by immunohistochemistry staining. HMB- 45 is considered to be the main histopathological biomarker for a HAML [1, 5]. Other biomarkers expressed in HAMLs include Melan $A$, vimentin, and SMA which were present in our case, as well as proto-oncogene c-Kit (CD 117), the latter being absent in our biopsy sample [1]. Metastatic melanoma is a potential differential diagnosis as this expresses both HMB-45 and Melan A [4].

There are a number of differences between hepatic and renal angiomyolipomas. HAMLs demonstrate a preponderance of the epitheloid smooth muscle cell component, as opposed to renal angiomyolipomas, which generally contain spindle-shaped muscle cells. The type of muscle cell present defines the degree and intensity of immunostaining of the biomarkers HMB-45, and Melan-A. These biomarkers are more strongly and more diffusely expressed in epitheloid cells and are therefore more suggestive of HAMLs [9].

In renal HAMLs, in view of the non-specific criteria to differentiate between benign and malignant lesions, these are usually surgically resected and followed-up thereafter [7].

\section{References}

1 Du S, Li Y, Mao Y, Sang X, Lu X, Wang W, et al. Diagnosis and treatment of hepatic angiomyolipoma. Hepatobiliary Surg Nutr. 2012 Dec;1(1):19-24.

2 Horvat N, Nikolovski I, Long N, Gerst S, Zheng J, Pak LM, et al. Imaging features of hepatocellular carcinoma compared to intrahepatic cholangiocarcinoma and combined tumor on MRI using liver imaging and data system (LI-RADS) version 2014. Abdom Radiol (NY). 2018 Jan;43(1):169-78.
The non-specific radiological findings as well as the varying histological composition of HAMLs make them difficult to distinguish from other liver tumors. Furthermore, surgical resection is the treatment of choice for a liver HAML in view of its malignant potential, risk of bleeding (size-related), and possibility of tumor rupture [1].

\section{Statement of Ethics}

The authors have no ethical conflicts to disclose. The subject has given informed consent to publish his case, including publication of images.

\section{Conflict of Interest Statement}

The authors have o conflicts of interest to disclose.

\section{Funding Sources}

The authors did not receive any funding.
3 Kubo H, Yamazaki H, Okada T, Takahashi Y, Nishi Y, Yokomori H. Primary hepatic angiomyolipoma: immunohistochemistry and electron microscopic observations: a case report. J Med Case Reports. 2017 Mar;11(1):76.

4 Mann SA, Saxena R. Differential diagnosis of epithelioid and clear cell tumors in the liver. Semin Diagn Pathol. 2017 Mar;34(2):183-91.

5 Yang X, Li A, Wu M. Hepatic angiomyolipoma: clinical, imaging and pathological features in 178 cases. Med Oncol. 2013 Mar; 30(1):416

6 Kubajak C, Lui SK, Vilchez V, Cartwright T, Ayoob A, Lee E, et al. Perivascular epitheloid Cell Tumor: A rare liver primary of mesenchymal origin. Surgery Curr Res. 2016;6(5): 275 .
7 Kamimura K, Nomoto M, Aoyagi Y. Hepatic angiomyolipoma: diagnostic findings and management. Int J Hepatol. 2012;2012: 410781.

8 Zhong DR, Ji XL. Hepatic angiomyolipomamisdiagnosis as hepatocellular carcinoma: A report of 14 cases. World J Gastroenterol. 2000 Aug;6(4):608-12.

9 Makhlouf HR, Ishak KG, Shekar R, Sesterhenn IA, Young DY, Fanburg-Smith JC. Melanoma markers in angiomyolipoma of the liver and kidney: a comparative study. Arch Pathol Lab Med. 2002 Jan;126(1):49-55. 